\title{
VECTOR FIELDS AND RICCI CURVATURE
}

\section{S. BOCHNER}

We shall prove theorems on nonexistence of certain types of vector fields on a compact manifold with a positive definite Riemannian metric whose Ricci curvature ${ }^{1}$ is either everywhere positive or everywhere negative. Actually we shall have some relaxations of the requirements both as to curvature and as to compactness. We shall deal with real spaces with a customary metric and with complex analytic spaces with an Hermitian metric. In the latter case we shall impose on the metric a certain restriction, first explicitly stated by E. Kaehler, ${ }^{2}$ which will be quite indispensable to our argument. In order to elucidate the rôle of this restriction we shall include a systematic introduction to the theory of Hermitian metric.

For positive curvature we shall have the theorem that on a compact space there exists no vector field for which the divergence and curl both vanish. In the complex case there exists no vector field whatsoever whose covariant components are analytic functions in the complex parameters. If we only assume that the curvature is nonnegative, then there are some "exceptional" vector fields in directions of spatial flatness. A principal result will be the following theorem on meromorphic functions. If a complex space with positive curvature is covered by a finite number of neighborhoods, if a meromorphic functional element is defined in each neighborhood, and if the difference of any two meromorphic elements is holomorphic wherever the elements overlap, then there exists one meromorphic function on the space which differs by a holomorphic function from each meromorphic element given. In a previous paper ${ }^{3}$ this conclusion was drawn in the

Received by the editors June 28, 1946; published with the invited addresses for reasons of space and editorial convenience.

1 Also called mean curvature; the definition will be restated later in the text. Interesting facts relating Ricci curvature to Riemann curvature have been given by T. Y. Thomas, On the variation of curvature in Riemann spaces of constant mean curvature, Annali di Matematica Pura ed Applicata (4) vol. 13 (1935) pp. 227-238, and New theorems on Riemann-Einstein spaces, Rec. Math. (Mat. Sbornik) N.S. vol. 3 (1938) pp. 331-340. Also, for the application of the Laplacean on compact Riemannian spaces see T. Y. Thomas, Some applications of Green's theorem for compact Riemann spaces, Tôhoku Math. J. vol. 46 (1940) pp. 261-266.

2 Ueber eine bemerkenswerte Hermitische Metrik, Abh. Math. Sem. Hamburgischen Univ. vol. 9 (1933) pp. 173-186; see also S. Chern, Characteristic classes of Hermitian manifolds, Ann. of Math. vol. 47 (1946) pp. 85-121, especially pp. 109-112.

${ }^{3} \mathrm{~S}$. Bochner, Analytic and meromorphic continuation by means of Green's formula, Ann. of Math. vol. 44 (1943) pp. 652-673, especially p. 672, Theorem 15. 
case of a multi-torus; however, in that case, due to the flatness of the space, some necessary and sufficient conditions on the meromorphic elements had to be added. These conditions correspond to the "exceptional" vector fields mentioned before, and they do not occur if the curvature is strictly positive.

For negative curvature our theorem will in the main be a generalization of a theorem of Schwarz and M. Noether ${ }^{4}$ from one to several complex variables. It states that on an algebraic curve of genus $p>1$ there is no one-parametric group of rational transformations. In other words, on such a compact two-dimensional surface in one complex variable there exists no continuous group of analytic homeomorphisms. Now, the universal covering surface of such a surface can be identified with the unit circle $|z|<1$ and thus endowed with a hyperbolic metric of (constant) negative curvature. Thus we can express the theorem of Schwarz and Noether in the following second version. If a "real" compact two-dimensional space with a Riemannian metric has (constant) negative curvature then there exists no continuous group of conformal (that is, angle-preserving) transformations into itself. Corresponding to these two versions we shall have two generalizations to several variables. The complex version will be that if a compact complex space in $n$ variables with a suitable Hermitian metric has negative Ricci curvature (not necessarily constant), then there exists no continuous group of complex homeomorphisms. The "real" version will be less satisfactory but rather illuminating. We shall first show that for negative curvature in $n$ variables there are no groups of motions. A motion preserves not only angles but also the lengths. In order to obtain a theorem on transformations which distort the length we shall have to add an assumption on the nature of the distortion. However, this assumption will be automatically fulfilled for $n=2$, and thus technically a proper generalization will be obtained. We shall also have "exceptional" groups in case the curvature is only nonpositive, and we shall have a further statement in case the given compact space is a minimal variety in some higher-dimensional space of nonpositive curvature.

Finally we shall have some converse theorems, but only locally. A converse theorem states that if there are sufficiently many vector fields of one kind or another then the curvature is negative or positive respectively. Locally, a gradient field is a scalar function from which it is derived, and several gradient fields is therefore a mapping into

${ }^{4}$ H. A. Schwarz, Gesammelte Abhandlungen, vol. 2, pp. 285-291; M. Noether, Math. Ann. vol. 20 (1882) pp. 59-62 and vol. 21 (1883) pp. 138-140. See also A. Hurwitz, Mathematische Werke, vol. 1, pp. 241-259. 
Euclidean space. Correspondingly we shall have the following theorems on complex space. Any piece of $m$-dimensional surface in $n$-dimensional Euclidean space has negative curvature, but the presence of sufficiently many contravariant vector fields in commutation gives rise to a fundamental tensor whose curvature is positive.

The most interesting problem in this connection is the problem of constructing a negative (hyperbolic) curvature from the existence of a noncommutative transitive group of analytic homeomorphisms on a piece of Euclidean or other space. This problem will not be approached at all in the present context.

\section{REAL SPACES}

1. Lemmas. Let $S$ be an $n$-dimensional coordinate space with a fixed positive definite Riemannian metric

$$
d s^{2}=g_{i j} d x_{i} d x_{j} .
$$

The space is of differentiability class $C^{3}$, and all scalars, vectors and tensors belong to class $C^{2}$. For any scalar $\phi$ we can form the Laplacean

$$
\Delta \phi \equiv g^{i j} \phi, i, j \equiv \frac{1}{g^{1 / 2}} \frac{\partial}{\partial x_{i}}\left(g^{1 / 2} g^{i j} \frac{\partial \phi}{\partial x_{j}}\right)
$$

and we shall apply it so the square length

$$
\phi=\xi^{i} \xi_{i} \equiv g^{i i j} \xi_{i} \xi_{j} \equiv g_{i j} \xi^{i} \xi^{i},
$$

where $\left\{\xi_{i}\right\}$ is an arbitrary vector field on all of $S$. We shall next have a definition.

Definition. A vector $\xi_{i}$ on $S$ is called a restrained vector, if either

$$
\Delta \phi\left(P_{0}\right)<0
$$

at some point $P_{0}$ in $S$, or

$$
\phi(P) \equiv \text { constant }
$$

throughout $S$.

This definition will be justified by the following theorem.

LEMMA 1. On a compact space $S$ every vector is restrained relative to every metric.

On a general $S$ a vector is restrained if its length in some metric attains a (relative) maximum in $S$, and thus in particular if the length is less than $\epsilon$ outside some compact subset depending on $\epsilon$.

Proof. The compact case can be dealt with by a "global" argument, namely Stokes' formula 


$$
\int\left(\phi \cdot \Delta \phi+g^{i j} \phi, i \phi, j\right) d v=0 .
$$

In fact, if $\phi \geqq 0, \Delta \phi \geqq 0$, then (5) can hold only if $\phi_{, i} \equiv 0$, that is, $\phi \equiv$ constant, as claimed. But there is also a "local" argument which implies the lemma in its entirety. This is the following theorem of E. Hopf. ${ }^{5}$ If $\Delta \phi \geqq 0$ in a neighborhood $T$, and if there exists a point $P_{0}$ in $T$ such that $\phi(P) \leqq \phi\left(P_{0}\right)$ for all $P$ in $T$, then $\phi(P) \equiv \phi\left(P_{0}\right)$ in $T$. We now start from the formula

$$
\xi_{i, j, k}-\xi_{i, k, j}-\xi_{l} R_{i j k}^{l}=0
$$

where $R_{i j k}^{l}$ is the curvature tensor. The Ricci tensor arises by contraction,

$$
R_{i j}=R_{i j k}^{k}=g^{l k} R_{l i j k}=R_{i j k}^{l},
$$

and it has the following interpretation. ${ }^{6}$ If for a given point we consider $n$ orthogonal unit vectors $\eta_{(m)}^{i}, m=1, \cdots, n$, which are cooriented with $x_{1}, \cdots, x_{n}$, and if we denote by $\kappa_{p q}$ the curvature of the geodesic surface element which is determined by $\eta_{(p)}^{i}$ and $\eta_{(q)}^{d}$, then the sum

$$
\kappa_{11}+\kappa_{12}+\cdots+\kappa_{1 n}
$$

is given by

$$
-R_{i j} \eta_{(1)}^{i} \eta_{(1)}^{j},
$$

thus being dependent only on $\eta_{(1)}^{i}$ itself. We shall call it the Ricci curvature in the direction $\eta_{(1)}^{i}$. The curvature is called positive if it is strictly positive in all directions.

Lemma 2. The quantity

$$
2^{-1} \Delta \phi=2^{-1} g^{r s}\left(g^{a b} \xi_{a} \xi_{b}\right)_{, r, s}=g^{r s} g^{a b} \xi_{a, r} \xi_{b, s}+g^{r s} g^{a b} \xi_{a, r, s} \xi_{b}
$$

has the value

$$
g^{r s} g^{a b} \xi_{a, r} \xi_{b, s}-R^{l m} \xi_{l} \xi_{m}+A
$$

where the remainder term is

- Elementare Bemerkungen ueber die Loesungen partieller Differentialgleichungen zweiter Ordnung vom elliptischen Typus, Preuss. Akad. Wiss. Sitzungsber. vol. 19 (1927) pp. 147-152.

${ }^{6}$ See L. P. Eisenhart, Riemannian geometry, p. 113. We have adopted Eisenhart's normalization throughout, and this explains why positive Ricci curvature corresponds to a negative Ricci quadratic form. 


$$
A=g^{r s} g^{a b}\left(\xi_{a, r, s}-\xi_{r, a, s}+\xi_{r, s, a}\right) \xi_{b} ;
$$

it also has the value

$$
g^{r s} g^{a b} \xi_{a, r} \xi_{b, s}+R^{l m} \xi_{l} \xi_{m}+B
$$

where

$$
B=g^{r s} g^{a b}\left(\xi_{a, r, s}+\xi_{r, a, s}-\xi_{r, s, a}\right) \xi_{b} .
$$

In fact, from (6) we obtain

$$
g^{r a} g^{a b}\left(\xi_{r, 8, a}-\xi_{r, a, s}\right) \xi_{b}-g^{a b} R_{a \xi_{l}}^{l} \xi_{b}=0 .
$$

If we add this to (8) we obtain (9), and if we subtract it from (8) we obtain (11).

2. Positive curvature. Since the term

$$
g^{r s} g^{a b} \xi_{a, r} \xi_{b, s}
$$

is positive for arbitrary quantities $\xi_{i, j}$ except in the case where the latter are all 0 , we first obtain the following conclusion.

THEOREM 1. The remainder term $A$ is obviously 0 whenever

$$
\xi_{i, j}=\xi_{j, i} \text { and } g^{i j} \xi_{i, j}=0,
$$

that is,

$$
\operatorname{curl} \xi=0 \text { and } \operatorname{div} \xi=0 \text {, }
$$

or more generally, whenever

$$
\left(\xi_{i, j}-\xi_{j, i}\right)_{, k}=0 \text { and } \quad\left(g^{i j \xi_{i, j}}\right)_{, k}=0 .
$$

Therefore, there exists no restrained vector field which satisfies relation (16) and

$$
-R^{i j} \xi_{i} \xi_{j} \geqq 0
$$

unless we have

$$
-R^{i i \xi_{i} \xi_{j}}=0, \quad \xi_{i, j}=0 .
$$

Thus, the only exceptions are fields of parallel vectors in directions of Ricci-flatness, and they cannot arise, for instance, if the space as such has positive Ricci curvature throughout.

A vector field for which $\xi_{i, j}=\xi_{j, i}$ is a local gradient field and can be described in the following way. There exists a covering of the space by a system of neighborhoods $\left\{U^{p}\right\}$, which for a compact space is finite, and a scalar $f^{(p)}(x)$ on each $U^{p}$, such that 


$$
f^{(p)}(x)-f^{(x)}(x)=\text { constant }
$$

whenever two neighborhoods $U^{p}, U^{a}$ intersect. And $\xi_{i}$ is defined as

$$
\xi_{i}=\frac{\partial}{\partial x_{i}} f^{(p)}(x)
$$

on $U^{p}$. The second part of (14) amounts to assuming that each $f^{(p)}(x)$ shall in its neighborhood be a solution of the Laplacean

$$
\Delta f=0,
$$

and our theorem states in particular that on a space of positive Ricci curvature every $f^{(p)}(x)$ is itself constant. It should be noted that this conclusion is trivial if $S$ is simply connected, or if some simply connected covering space of $S$ is compact, since in this case the system of equations (20) can be solved by one function $f(x)$ in $S$; and since its Laplacean vanishes, it is a constant throughout.

3. Negative curvature. We now turn to an analysis of the expression (11) and we shall first have a detailed lemma on the influence of the remainder term $B$.

LEMMA 3. The remainder term $B$ vanishes if

$$
\xi_{i, j}+\xi_{j, i}=0,
$$

or more generally if

$$
\left(\xi_{i, j}+\xi_{j, i}\right)_{, k}=0 .
$$

Thus it vanishes if the contravariant components $\xi^{i}$ are an infinitesimal generator of a one-parametric group of motions, that is, metric-preserving homeomorphisms, or, more generally, of affine collineations.

We have $B \geqq 0$, if there exists a scalar function $\lambda(x)$ such that

$$
\xi_{i, j}+\xi_{j, i}=\lambda g_{i j},
$$

that is, if $\xi^{i}$ represents a one-parametric group of angle-preserving (conformal) homeomorphisms, provided we also have

$$
(1-n / 2) g^{i j} \lambda_{i} \xi_{j} \geqq 0,
$$

the latter condition being automatically fulfilled for $n=2$.

Proof. If we multiply (23) by $g^{i j}$, then due to the symmetry of $g^{i j}$ we obtain

$$
g^{r s} \xi_{r, s, a}=0,
$$

and thus (23) implies $B=0$. On any differentiable manifold, with or 
without a Riemannian metric, a vector $\xi^{i}$ describes a local group of differentiable homeomorphisms. If a metric is given we can form the covariant components $\xi_{i},(22)$ is the necessary and sufficient condition for the transformations to be motions, ${ }^{7}$ and (23) for affine collineations.

Condition (24) expresses preservation of angles. If we differentiate it we obtain

$$
\xi_{i, j, k}+\xi_{j, i, k}=\lambda_{, k} g_{i j}
$$

and hence

$$
g^{r s} g^{a b}\left(\xi_{a, r, s}+\xi_{r, a, s}\right) \xi_{b}=g^{s b} \lambda_{, 8} \xi_{b} .
$$

If, however, we multiply (26) by $g^{i j} g^{k b} \xi_{b}$ we obtain

$$
-g^{r s} g^{a b} \xi_{r, s, a} \xi_{b}=-(n / 2) g^{s b} \lambda_{s} \xi_{b}
$$

and thus (25) is equivalent with $B \geqq 0$.

THEOREM 2. There exists no restrained group of motions $\left\{\xi^{i}\right\}$ for which

$$
-R_{i j} \xi^{i} \xi^{i} \leqq 0
$$

unless we have

$$
\xi_{i, j}=0, \quad R_{i j} \xi^{i} \xi^{i}=0,
$$

that is, unless it is a group of translations along Ricci-flat geodesics.

For $n=2$, the same conclusion holds for conformal mappings $\left\{\xi^{i}\right\}$, and for $n \geqq 3$ it will hold under the additional assumption (25).

4. Almost periodic vector fields. Take a compact space $S$ and its universal covering space $T$. If $S$ has negative curvature, $T$ most likely will not be compact. Introduce on $T$ the group $\Gamma$ of automorphisms for which $S$ is the set of co-sets, and denote by $S_{0}$ a fundamental domain in $T$. It can be chosen compact. Every scalar or tensor function on $S$ gives rise to such a function on $T$ which is "periodic," that is, invariant under $\Gamma$. In particular the tensor $g_{i j}$ can be periodically extended. Now take a vector field $\xi_{i}$ on $T$ which, though not necessarily periodic, is together with its second derivatives almost periodic with respect to $\Gamma$. This means that if $\left\{\gamma_{p}\right\}$ is a sequence in $\Gamma$ then there exists a sub-sequence $\left\{\gamma_{q}\right\}$ such that the sequence of vector fields $\xi_{i}\left(\gamma_{q} P\right)$ and their second derivatives is uniformly convergent in $T$.

\footnotetext{
${ }^{7}$ Eisenhart, p. 234.
} 
THEOREM 3. Any almost periodic vector field $\xi_{i}$ on the covering space $T$ of $S$ is restrained in $T$, and thus Theorems 1 and 2, and similar theorems, apply to such vector fields in $T$.

Proof. Due to its almost periodicity, the square length $\phi(P)$ of $\xi_{i}$ is bounded and uniformly continuous, and there exists a sequence of points $P_{p}$ such that

$$
\phi\left(P_{p}\right) \rightarrow \sup _{P} \phi(P)
$$

Each $P_{p}$ lies in some image $\gamma_{p}\left(S_{0}\right)$ of $S_{0}$, and owing to the compactness of $S_{0}$ there exists a subsequence $\gamma_{q}$ such that the sequence of translated functions $\phi\left(\gamma_{q} P\right)$ and their second derivatives will converge uniformly in $T$ towards a function $\Phi(P)$ which assumes its maximum in an interior point of $T$. Now, due to the periodicity of $g_{i j}$, we have

$$
\Delta \phi(P)=\Delta \phi\left(\gamma_{q} P\right)=\Delta \Phi(P),
$$

and thus $\Delta \phi \geqq 0$ implies $\Delta \Phi \geqq 0$. By Lemma 1 , we therefore have $\Phi=$ const. However this implies $\phi=$ const., and thus $\xi_{i}$ is restrained.

5. Minimal varieties. If $V_{n}$ is a subspace of any space $V_{n+1}$ whose Riemann-Christoffel tensor vanishes, then for the Ricci tensor of $V_{n}$ we have $R_{j k}=g^{i l}\left(b_{i k} b_{j l}-b_{i l} b_{j k}\right)$ where $b_{i j}$ are the coefficients of the second differential form. ${ }^{8}$ If $V_{n}$ is a minimal hypersurface of $V_{n+1}$, then $g^{i j} b_{i j}=0,{ }^{9}$ and therefore

$$
R_{j k} \xi^{i} \xi^{k}=g^{i l} \eta_{i} \eta_{l}
$$

where

$$
\eta_{i}=b_{i k} \xi^{k}
$$

Thus the vanishing of (29) implies the vanishing of (30), and the vanishing of the latter for a group $\left\{\xi^{k}\right\}$ means that the orbits are asymptotic lines on $V_{n} \cdot{ }^{10}$ However, any geodesic line of $V_{n}$ which is asymptotic on $V_{n}$ is a geodesic of $V_{n+1}$ itself. The same reasoning applies to a minimal variety in a space $V_{m}$ of any higher dimension $m \geqq n+1$, since we have in general

$$
R_{j k} \xi^{j} \xi^{k}=\sum_{\sigma=1}^{m-n} g^{i j} b_{i k}^{(\sigma)} \xi^{k} b_{j l}^{(\sigma)} \xi^{l}
$$

\footnotetext{
${ }^{8}$ Eisenhart, p. 190.

${ }^{9}$ Eisenhart, p. 178.

${ }^{10}$ Eisenhart, p. 167.
} 
Thus we obtain the following theorem.

THEOREM 4. If the space $V_{n}$ is a minimal variety in a space $V_{m}$, $m \geqq n+1$, of vanishing Riemann curvature, and if there exists a oneparametric group of motions on $V_{n}$, then the orbits are geodesics in the enveloping $V_{m}$ which are also asymptotic lines on $V_{n}$.

\section{HeRmitian METRIC}

6. Power series. We take independent complex variables

$$
t_{1}, \cdots, t_{m}
$$

and we consider power series in these variables with complex coeffcients, each series converging in some (not fixed) neighborhood of the origin. Every "function," that is scalar, scalar density or any component of a tensor shall be such a series.

A "transformation"

$$
t_{i}^{\prime}=\phi_{i}\left(t_{1}, \cdots, t_{m}\right), \quad i=1, \cdots, m,
$$

shall be given by functions $\phi_{i}$ which vanish at the origin, in order to allow unrestricted formation of the product of two transformations, and the Jacobian

$$
\frac{\partial\left(t_{1}^{\prime}, \cdots, t_{m}^{\prime}\right)}{\partial\left(t_{1}, \cdots, t_{m}\right)}
$$

shall be not equal to 0 at the origin. The totality of such transformations forms a group, and we consider an arbitrary fixed subgroup $\Gamma$ of the group. The elements of $\Gamma$ shall be termed allowable transformations and the resulting quantities $t_{i}^{\prime}$ allowable variables. We can now define as usual vectors and tensors

$$
\xi_{i}, \xi^{i}, \xi_{i}{ }^{k}{ }^{2} \ldots,
$$

with upper and lower indices; also, contraction of an upper and lower index is again permitted. A symmetric affine connection $\Gamma_{j k}$ is again a system of functions with the customary rules of transformation. It gives rise to a mixed tensor

$$
R_{i j k}^{l}=\frac{\partial}{\partial t_{j}} \Gamma_{i k}^{l}-\frac{\partial}{\partial t_{k}} \Gamma_{i j}^{l}+\Gamma_{i k}^{m} \Gamma_{m j}^{l}-\Gamma_{i j}^{m} \Gamma_{m k}^{l}
$$

with which we can form the Ricci tensor

$$
R_{i j}=R_{i j k}^{k}
$$


it also gives rise to covariant differentiation of arbitrary tensors and tensor densities as usual. For instance

$$
\begin{aligned}
\xi_{i, j} & =\frac{\partial \xi_{i}}{\partial t_{j}}-\xi_{k} \Gamma_{i j}^{k}, \\
\xi_{i, j}^{i} & =\frac{\partial \xi^{i}}{\partial t_{j}}+\xi^{k} \Gamma_{k j .}^{i}
\end{aligned}
$$

Also derivatives of sums, products and contractions are to be formed as usual.

We next introduce a fundamental tensor $g_{i j}$. For the present we only assume that it is symmetric

$$
g_{i j}=g_{j i},
$$

and that the determinant

$$
g=\left\|g_{i j}\right\|_{i, j=1, \cdots, m},
$$

is not equal to 0 at the origin. We emphasize that each component $g_{i j}$ is a complex power series in the complex variables (31) and that the symmetry condition (37) is not at all an "Hermitian" property. There exist functions $g^{i j}$ which are solutions of $g^{i j} g_{i k}=\delta_{k}^{i}$, and they are a symmetric tensor. The expressions

$$
\Gamma_{j k}^{i}=2^{-1} g^{i l}\left(\frac{\partial g_{l k}}{\partial t_{j}}+\frac{\partial g_{j l}}{\partial t_{k}}-\frac{\partial g_{j k}}{\partial t_{l}}\right)
$$

are an affine connection, and the tensors $g_{i j}, g^{i j}$ can be used for pulling indices up and down in a manner compatible with covariant differentiation. Also

$$
g_{i j, k}=0, \quad g^{i j}, k=0,
$$

and ${ }^{11}$

(40) $R_{i j}=2^{-1} \frac{\partial^{2}}{\partial t_{i} \partial t_{j}} \log \frac{g}{C_{0}}-\frac{\partial}{\partial t_{k}} \Gamma_{i j}^{k}+\Gamma_{i k}^{m} \Gamma_{m j}^{k}-2^{-1} \Gamma_{i j}^{m} \frac{\partial}{\partial t_{m}} \log \frac{g}{C_{0}}$, where $C_{0}$ is the value of the determinant $g$ at the origin. Also

$$
R_{j k l}^{i}+R_{k l j}^{i}+R_{l j k}^{i}=0
$$

and

$$
R_{i j k l}=-R_{j i k l}=-R_{k l j i}
$$

\footnotetext{
"w Eisenhart, p. 21.
} 
7. Self-adjointness. We now put $m=2 n$ and we replace the variables (31) by the symbols

$$
z_{1}, \cdots, z_{n} ; \bar{z}_{1}, \cdots, \bar{z}_{n} \text {. }
$$

Allowable transformations shall be those having the special form

$$
z_{\alpha}^{\prime}=\phi_{\alpha}\left(z_{1}, \cdots, z_{n}\right), \quad \bar{z}_{\alpha}^{\prime}=\psi_{\alpha}\left(\bar{z}_{1}, \cdots, \bar{z}_{n}\right),
$$

where for each $\alpha=1, \cdots, n$ the power series $\psi_{\alpha}\left(t_{1}, \cdots, t_{n}\right)$ arises from the power series $\phi_{\alpha}\left(t_{1}, \cdots, t_{n}\right)$ by replacing each coefficient by its conjugate complex value. Therefore, if each $\bar{z}_{\alpha}$ is conjugate to $z_{\alpha}$, then so is $\bar{z}_{\alpha}^{\prime}$ to $z_{\alpha}^{\prime}$, and therefore it will be consistent to assume henceforth that $z_{\alpha}$ are complex parameters and $\bar{z}_{\alpha}$ their conjugate values. However a function in general will continue to be a power series in all $2 n$ variables (43), or rather it will be a power series with complex coefficients in the $2 n$ real components $x_{\alpha}, y_{\alpha} ; z_{\alpha}=x_{\alpha}+i y_{\alpha}$. Special scalar or tensor functions whose power series depend only on $\boldsymbol{z}_{\boldsymbol{\alpha}}$ will be termed "complex analytic" or "analytic" for short.

From now on italic indices $i, j, k, \cdots$ will run from 1 to $2 n$; Greek indices $\alpha, \beta, \cdots$ from 1 to $n$, and starred Greek indices will be $n$ units more, $\alpha^{*}=\alpha+n$. We shall also star italic indices to indicate a change by $n$, thus $i^{*}=i \pm n, j^{*}=j \pm n$.

Let $\zeta_{i}$ be a covariant vector. For a transformation (44) we have

$$
\zeta_{\alpha}^{\prime}=\zeta_{\beta} \frac{\partial z_{\beta}^{\prime}}{\partial z_{\alpha}}, \quad \zeta_{\alpha^{*}}^{\prime}=\zeta_{\beta^{*}} \frac{\partial \bar{z}_{\beta}^{\prime}}{\partial \bar{z}_{\alpha}} .
$$

Thus the $2 n$ components of $\zeta_{i}$ separate completely into the blocks $\zeta_{\alpha}$

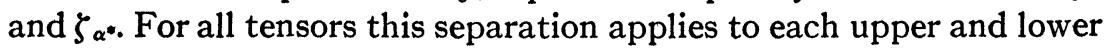
index independently of the others. For instance, $g_{i j}$ separates into the blocks $g_{\alpha \beta}, g_{\beta \alpha}, g_{\alpha \beta^{*}} g_{\alpha^{*} \beta}$, of $n^{2}$ components each.

We call a tensor self-adjoint if starring all indices simultaneously changes the value of a component into its conjugate:

$$
\zeta_{i^{*} k^{*} l^{*} \ldots}^{j^{*}}=\overline{\zeta_{i k l} \ldots}
$$

For a vector it means

$$
\zeta_{\alpha^{*}}=\bar{\zeta}_{\alpha}, \quad \zeta^{\alpha^{*}}=\overline{\zeta^{\alpha}},
$$

and for a symmetric tensor $g_{i j}$ it means

$$
\begin{aligned}
g_{\alpha \beta}=g_{\beta \alpha} & =\overline{g_{\alpha^{*} \beta^{*}}}=\overline{g_{\beta^{*} \alpha^{*}}}, \\
g_{\alpha \beta^{*}} & =g_{\beta^{*} \alpha}=\overline{g_{\alpha^{*} \beta}}=\overline{g_{\beta} \alpha^{*} .}
\end{aligned}
$$

A scalar shall be self-adjoint if it is real-valued. Self-adjointness is 
preserved under addition, multiplication and contraction. If $g_{i j}$ is self-adjoint, then the inverse $g^{i j}$, and $\Gamma_{j k}^{i}$ based on $g_{i j}$, are self-adjoint, and so are the Riemann and Ricci tensors. Furthermore it is preserved under covariant differentiation.

From now on all functions are assumed to be self-adjoint.

8. Restrictions on the fundamental tensor. The first substantial restriction on the fundamental tensor shall be

$$
g_{\alpha \beta}=0=g_{\alpha^{*} \beta^{*}}
$$

Thus only the "interacting" components $g_{\alpha \beta^{*}}=g_{\beta^{*} \alpha}$ can be not equal to 0 , and $g_{\alpha \beta^{*}}=\overline{g_{\alpha^{*} \beta}}$. We automatically also have

$$
g^{\alpha \beta}=0
$$

and (38) gives

$$
\begin{aligned}
\Gamma_{\beta \gamma}^{\alpha} & =2^{-1} g^{\alpha \epsilon^{*}}\left(\frac{\partial g_{\epsilon^{*} \gamma}}{\partial z_{\beta}}+\frac{\partial g_{\beta \epsilon^{*}}}{\partial z_{\gamma}}\right), \\
\Gamma_{\beta \gamma^{*}}^{\alpha} & =2^{-1} g^{\alpha \epsilon^{*}}\left(\frac{\partial g_{\beta \epsilon^{*}}}{\partial \bar{z}_{\gamma}}-\frac{\partial g_{\beta \gamma^{*}}}{\partial \bar{z}_{\epsilon}}\right), \\
\Gamma_{\beta^{*} \gamma^{*}}^{\alpha} & =0,
\end{aligned}
$$

and 5 other relations obtained by symmetry and self-adjointness. From (35) we now have

$$
\begin{gathered}
\zeta_{\alpha, \beta}=\frac{\partial \zeta_{\alpha}}{\partial z_{\beta}}-\zeta_{\epsilon} \Gamma_{\alpha \beta}^{e} \\
\zeta_{\alpha^{*}, \beta}=\frac{\partial \zeta_{\alpha}}{\partial \bar{z}_{\beta}}-\zeta_{\epsilon} \Gamma_{\alpha \beta^{*}}^{e}
\end{gathered}
$$

However we also want to have $\Gamma_{\beta \gamma^{*}}^{\alpha}=0$, and this will be obtained by Kaehler's restriction, which is:

$$
\frac{\partial g_{\alpha \gamma^{*}}}{\partial z_{\beta}}=\frac{\partial g_{\beta \gamma^{*}}}{\partial z_{\alpha}},
$$

and by adjointness also implies

$$
\frac{\partial g_{\gamma \alpha^{*}}}{\partial \bar{z}_{\beta}}=\frac{\partial g_{\gamma \beta^{*}}}{\partial \bar{z}_{\alpha}} .
$$

This is equivalent with assuming that there exists (locally) a scalar function $\phi(z, \bar{z})$ such that 


$$
g_{\alpha \beta^{*}}=\frac{\partial^{2} \Phi}{\partial z_{\alpha} \partial \bar{z}_{\beta}} .
$$

Self-adjointness demands that $\Phi$ shall be real-valued, and all these assumptions are indeed invariant under transformations of the type (43). We shall now list consequences of our restrictions.

\section{LEMma 4. We have}

$$
\zeta_{\alpha, \beta^{*}}=\frac{\partial \zeta_{\alpha}}{\partial \bar{z}_{\beta}}, \quad \zeta^{\alpha}, \beta^{*}=\frac{\partial \zeta^{\alpha}}{\partial \bar{z}_{\beta}} .
$$

In particular if the component $\zeta_{\alpha}$ is complex analytic, we have $\zeta_{\alpha, \beta^{*}}=0$, and if $\zeta^{\alpha}$ is complex analytic we have $\zeta^{\alpha}, \beta^{*}=0$.

More generally, if the indices $\alpha_{1}, \cdots, \alpha_{p}$ are unstarred, we have

$$
\zeta_{\alpha_{1}, \cdots, \alpha_{p}, \beta^{*}}=\frac{\partial \zeta_{\alpha_{1}, \cdots, \alpha_{p}}}{\partial \bar{z}_{\beta}} ; \quad \zeta^{\alpha_{1}, \cdots, \alpha_{p}, \beta^{*}}=\frac{\partial \zeta^{\alpha_{1}, \cdots, \alpha_{p}}}{\partial \bar{z}_{\beta}}
$$

For the appreciation of the lemma it should be noted that if the $n$ components $\zeta_{\alpha}$ are complex analytic the contravariant components

$$
\zeta^{\alpha}=g^{\alpha \beta^{*}} \xi_{\beta}, \quad \zeta^{\alpha^{*}}=g^{\alpha^{*} \beta} \zeta_{\beta}
$$

will depend on all $2 n$ variables (42) since the components $g^{\alpha \beta^{*}}$ so depend.

LEMma 5. For the Riemann tensor we have

$$
R_{\alpha \beta j k}=0 \text {. }
$$

More generally, due to (42), only those components of $R_{\text {lijk }}$ can be different from 0 for which, in both pairs of indices $(l, i)$ and $(j, k)$ simultaneously, one element in the pair is a Greek unstarred and the other a Greek starred index.

Proof. It suffices to prove that $R_{\beta j k}^{\alpha *}$ vanishes. From (33) we obtain for this component the value

$$
\frac{\partial}{\partial t_{j}} \Gamma_{\beta k}^{\alpha^{*}}-\frac{\partial}{\partial t_{k}} \Gamma_{\beta j}^{\alpha^{*}}+\Gamma_{\beta k}^{m} \Gamma_{m j}^{\alpha^{*}}-\Gamma_{\beta j}^{m} \Gamma_{m k}^{\alpha^{*}},
$$

and this vanishes since only the pure components $\Gamma_{\beta_{\gamma}}^{\alpha}$ and $\overline{\Gamma_{\beta \gamma}^{\alpha}}$ can be not equal to 0 .

LEMMA 6. We also have

$$
R_{\beta \gamma^{*} \delta}^{\alpha}=-R_{\delta \beta \gamma^{*}}^{\alpha}
$$


Proof. In the Bianchi identity

$$
R_{\beta \gamma^{\circ} \delta}^{\alpha}+R_{\gamma^{* \beta \delta}}^{\alpha}+R_{\delta \beta \gamma^{*}}^{\alpha}=0
$$

the middle term is 0 by Lemma 5 .

Lemma 7. For the Ricci tensor we have

$$
R_{\alpha \beta}=0 \text {, }
$$

$$
R_{\alpha \beta}=\frac{\partial^{2} \log |G|}{\partial z_{\alpha} \partial \bar{z}_{\beta}}
$$

where $G$ is the determinant

$$
G=\left\|g_{\alpha \beta}\right\|_{\alpha, \beta=1, \cdots, n} .
$$

Proof. (57) follows from

$$
R_{\alpha \beta^{*}}=g^{l k} R_{l \alpha \beta k}
$$

by Lemma 5, and (58) follows from (40).

9. Positive fundamental tensor. Finally, we make the assumption that the matrix $g_{\alpha \beta}$ is positive-definite. Consequently, for any vector $\zeta_{i}$ the "square length"

$$
\phi=g^{i_{i} \zeta_{i} \zeta_{j}}=2 g^{\alpha \beta^{*}} \zeta_{\alpha} \bar{\zeta}_{\beta}
$$

is non-negative. For the Laplacean we have

$$
\Delta \phi=g^{i j} \phi, i, j=g^{\alpha \beta^{*}} \phi_{, \alpha, \beta^{*}}+g^{\alpha^{*} \beta} \phi, \alpha^{*}, \beta,
$$

and by Lemma 4 this is

$$
2 g^{\alpha \beta *} \frac{\partial^{2} \phi}{\partial z_{\alpha} \partial \bar{z}_{\beta}} .
$$

Now, if we herein put $z_{\alpha}=x_{\alpha}+i y_{\alpha}$, then the resulting expression is a type of Laplacean in the variables $\left(x_{\alpha}, y_{\alpha}\right)$ to which Lemma 1 will apply.

If $\Phi$ is the function occurring in (55), the positiveness of the tensor is expressed by the relation

$$
\frac{\partial^{2} \Phi}{\partial z_{\alpha} \partial \bar{z}_{\beta}} \zeta^{\alpha} \overline{\zeta^{\beta}} \geqq 0,
$$

which is supposed to hold for every vector $\zeta^{\alpha}$. Also, we shall say that the Ricci curvature is nonpositive if $R_{\alpha \beta^{*}} \zeta^{\alpha} \zeta^{\beta^{*}} \geqq 0$, and on account of (58) this is 


$$
\frac{\partial^{2} \log G}{\partial z_{\alpha} \partial \bar{z}_{\beta}} \zeta^{\alpha} \overline{\zeta^{\beta}} \geqq 0,
$$

which is the same type of relation as (61) is. It is not hard to see that relations (61) and (62) must hold for arbitrary complex numbers $\zeta^{1}, \cdots, \zeta^{n}$. In particular, if we introduce a complex curve $z_{\alpha}=z_{\alpha}(\omega)$, $\omega=u+i v$, and $\zeta^{\alpha}=d z_{\alpha} / d \omega$, then we must have $\partial^{2} \phi / \partial \omega \partial \bar{\omega} \geqq 0$, that is, $\partial^{2} \phi / \partial u^{2}+\partial \phi^{2} / \partial v^{2} \geqq 0$. In other words the function $\Phi$ must be a subharmonic function on any analytic curve. Similarly in the case (62), the function $\log G$ must be subharmonic in the manner stated.

\section{COMPLEX SPACES}

10. Vector fields. A complex space is a coordinate space in $2 n$ real variables. Every basic neighborhood is the topological image of a neighborhood of the Euclidean space in $n$ complex parameters. If two basic neighborhoods intersect, then the two images of the intersection are mapped into each other by analytic functions

$$
z_{\alpha}^{\prime}=\phi_{\alpha}\left(z_{1}, \cdots, z_{n}\right), \quad \alpha=1, \cdots, n .
$$

It is easily seen that all concepts and results of Chapter II can be extended to a complex space in the large. In particular we assume the existence of a tensor $g_{i j}$ with all properties and restrictions as enumerated before. We define a restrained vector field as before, and on a compact space every vector field is again restrained.

LemMA 8. If the covariant components $\zeta_{\alpha}$ are complex analytic then for the scalar

$$
\phi=2 g^{\alpha \beta^{*}} \zeta_{\alpha} \zeta_{\beta^{*}}
$$

we have

$$
4^{-1} \Delta \phi=g^{\rho \sigma^{*}} g^{\alpha \beta^{*}} \zeta_{\alpha, \rho} \zeta \beta_{\beta^{*}, \sigma^{*}}-R^{\alpha \beta^{*}} \zeta_{\alpha} \zeta_{\beta^{*}}
$$

If however the contravariant components $\zeta^{\alpha}$ are analytic then we have

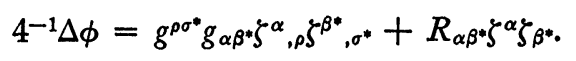

Proof. In the first case we have on basis of Lemmas 4 and 6

$$
4^{-1} \Delta \phi=g^{\rho \sigma^{*}}\left(g^{\alpha \beta^{*}} \zeta_{\alpha} \zeta_{\beta^{*}}\right)_{, \rho, \sigma^{*}}=g^{\rho \sigma^{*}} g^{\alpha \beta^{*}} \zeta_{\alpha, \rho} \zeta_{\beta^{*}, \sigma^{*}}+g^{\rho \sigma^{*}} g^{\alpha \beta^{*}} \zeta_{\alpha, \rho, \sigma^{*}} \zeta_{\beta^{*}} .
$$

Now

$$
\zeta_{\alpha, \rho, \sigma^{*}}=\zeta_{\alpha, \rho, \sigma^{*}}-\zeta_{\alpha, \sigma^{*}, \rho}=-\zeta_{\lambda} R_{\alpha, \sigma^{*}, \rho}^{\lambda}
$$

and by Lemma 6 this is 


$$
+\zeta_{\lambda} R_{\rho \alpha \sigma^{*}}^{\lambda}=-\zeta_{\lambda} R_{\rho \sigma^{*} \alpha}^{\lambda}
$$

Therefore

$$
g^{\rho \sigma^{*} \alpha \beta^{*}} \xi_{\alpha, \rho, 0}^{*} \zeta_{\beta}=-\zeta_{\lambda} g^{\alpha \beta^{*}} R_{\alpha}^{\lambda} \zeta_{\beta^{*}}=-\zeta_{\lambda} \zeta_{\beta^{*} \cdot R^{\lambda \beta^{*}}}
$$

In the second case we have, as claimed,

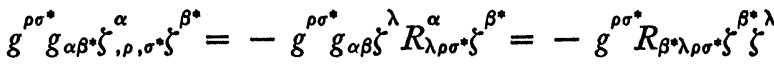

$$
\begin{aligned}
& =-g^{\rho \sigma^{*}} R_{\beta^{*} \sigma^{*} \lambda \rho} \zeta^{\beta^{*} \zeta^{\lambda}}=R_{\beta \lambda} \zeta^{\beta^{*} \lambda} \zeta^{\lambda} \text {. }
\end{aligned}
$$

From Lemma 8 we obtain directly the following theorem, the first half of which is an unqualified extension of the theorem of Schwarz and Noether from one to several complex variables.

THEOREM 5. On a compact space of negative Ricci curvature there exists no one-parametric group of analytic homeomorphisms whatsoever. More specifically, if on any space in a restricted vector field the contravariant components $\zeta^{\alpha}$ are complex analytic and $-R_{\alpha \beta} \zeta^{\alpha} \zeta^{\beta^{*}} \leqq 0$ holds, then we of necessity have $\zeta^{\alpha},{ }_{\beta}=0$ and $R_{\alpha \beta} \zeta^{\alpha} \zeta^{\beta^{*}}=0$.

If however the covariant components $\zeta_{\alpha}$ are analytic then the inequality $-R^{\alpha \beta^{*}} \zeta_{\alpha} \zeta_{\beta_{*}} \geqq 0$ must be a strict equality and it implies $\zeta_{\alpha, \beta}=0$.

It should be noted that the second half of the theorem is not restricted to local gradient fields as was Theorem 1.

It is very easy to extend Theorem $\mathbf{5}$ from vectors to tensors.

THEOREM 6. If a compact space has positive Ricci curvature then there exists no analytic covariant tensor $\zeta_{\alpha_{1} \ldots \alpha_{p}}$ whatsoever, and for negative curvature there exists no analytic contravariant tensor whatsoever.

Proof. For instance, in the case $p=2$, if we put

we obtain

$$
\phi=4 g^{\alpha_{1} \beta^{*}} g^{\alpha_{2} \beta^{*}} \zeta_{\alpha_{1} \alpha_{2}} \zeta_{\beta^{*} \beta^{*}{ }_{2}}
$$

$$
8^{-1} \Delta \phi=g^{\rho \sigma^{*}} g^{\alpha_{1} \beta_{2}} g^{\alpha_{2} \beta_{1}} \zeta_{\alpha_{1} \alpha_{2}, \rho} \zeta_{\beta^{*} \beta^{*}, \sigma^{*}}+A
$$

where

However,

$$
A=g^{\rho \sigma^{*}} g^{\alpha_{1} \beta_{1} g_{2} \beta_{2} \beta_{2}} \zeta_{\alpha_{1} \alpha_{2}, \rho, \sigma^{*}} \zeta_{\beta^{*} \beta_{2} \beta_{2}} .
$$

$$
\begin{aligned}
\zeta_{\alpha 1 \alpha_{2}, \rho, \sigma^{*}} & =\zeta_{\alpha 1 \alpha_{2}, \rho, \sigma^{*}}-\zeta_{\alpha_{1} \alpha_{2}, \sigma^{*}, \rho}=-\zeta_{\lambda \alpha_{2}} R_{\alpha_{11 \sigma^{*} \rho}}^{\lambda}-\zeta_{\alpha_{1} \lambda} R_{\alpha_{2} \sigma^{*} \rho}^{\lambda} \\
& =-\zeta_{\lambda \alpha_{2}} R_{\rho \alpha_{1} \sigma^{*}}^{\lambda}-\zeta_{\alpha_{1} \lambda} R_{\rho \alpha_{2} \sigma^{*} .}^{\lambda}
\end{aligned}
$$

Therefore

$$
A=-\zeta_{\lambda \alpha_{2}} \zeta_{\beta_{1} \beta^{*}{ }_{2}} R^{\lambda \beta^{*}} g^{\alpha_{2} \beta_{2}}-\zeta_{\alpha_{1} \lambda} \zeta_{\beta^{*} \beta_{2}} R^{\lambda \beta^{*}} g^{\alpha_{1} \beta^{*}},
$$

and the conclusion follows. 
11. Meromorphic functions. We consider a (self-adjoint) vector field which in the neighborhood of every point can be represented in the form

$$
\zeta_{\alpha}=\frac{\partial f}{\partial z_{\alpha}}, \quad \zeta_{\alpha^{*}}=\bar{\zeta}_{\alpha}=\frac{\partial \bar{f}}{\partial \bar{z}_{\alpha}} .
$$

It is not a local gradient field in the proper sense unless $f=\bar{f}$; that is, if $f$ is real-valued, and in our application it will definitely not be so. We introduce the associate vector

$$
\eta_{\alpha}=\frac{\partial \bar{f}}{\partial z_{\alpha}}, \quad \eta_{\alpha^{*}}=\frac{\partial f}{\partial \bar{z}_{\alpha}} .
$$

These two vectors are not subject to Lemma 4; however they have the following alternate properties

$$
\begin{aligned}
\zeta_{\alpha, \beta}=\zeta_{\beta, \alpha}, & \zeta_{\alpha^{*}, \beta^{*}}=\zeta_{\beta^{*}, \alpha^{*}}, \\
\eta_{\alpha, \beta}=\eta_{\beta, \alpha}, & \eta_{\alpha^{*}, \beta^{*}}=\eta_{\beta^{*}, \alpha^{*}} \\
\zeta_{\alpha, \beta^{*}}=\eta_{\beta^{*}, \alpha}, & \zeta_{\alpha, \beta}=\eta_{\beta, \alpha^{*} .}
\end{aligned}
$$

For $\phi=2 g^{\alpha \beta^{*}} \zeta_{\alpha} \zeta_{\beta^{*}}$ we obtain

$$
8^{-1} \Delta \phi=A+B+C
$$

where

$$
\begin{aligned}
& A=g^{\rho \sigma^{*}} g^{\alpha \beta^{*}}\left(\zeta_{\alpha, \rho} \zeta_{\beta^{*}, \sigma^{*}}+\zeta_{\alpha, \sigma^{*}} \zeta_{\beta^{*}, \rho}\right), \\
& B=g^{\rho \sigma^{*}} g^{\alpha \beta^{*}} \zeta_{\alpha, \rho, \sigma^{*}} \zeta_{\beta^{*}}, \\
& C=g^{\rho \sigma^{*}} g^{\alpha \beta^{*}} \zeta_{\alpha} \zeta_{\beta^{*}, \rho, \sigma^{*}} .
\end{aligned}
$$

If we substitute $\zeta_{\alpha, \rho, \sigma^{*}}=\zeta_{\rho, \alpha, \sigma^{*}}=\left(\zeta_{\rho, \alpha, \sigma^{*}}-\zeta_{\rho, \sigma^{*}, \alpha}\right)+\zeta_{\rho, \sigma^{*}, \alpha}=\zeta_{\rho, \sigma^{*}, \alpha}$ $-\zeta_{\lambda} R_{\rho \alpha \sigma^{*}}$ we obtain

$$
B=g^{\alpha \beta^{*}}\left(g^{\rho \sigma^{*}} \zeta_{\rho, \sigma^{*}}\right), \alpha \zeta_{\beta^{*}}-R^{\lambda \beta^{*}} \zeta_{\lambda} \zeta_{\beta^{*}},
$$

and if we put

$$
\zeta_{\beta^{*}, \rho, \sigma^{*}}=\eta_{\rho, \beta^{*}, \sigma^{*}}=\eta_{\rho, \sigma^{*}, \beta^{*}}+\left(\eta_{\rho, \beta^{*}, \sigma^{*}}-\eta_{\rho, \sigma^{*}, \beta^{*}}\right)=\eta_{\rho, \sigma^{*}, \beta}+\zeta_{\lambda} \hat{R}_{\rho \beta^{*} \sigma^{*}}
$$

we obtain by Lemma 5

$$
C=g^{\alpha \beta^{*}}\left(g^{\rho \sigma^{*}}, \eta_{\rho, \sigma^{*}}\right)_{, \beta} \zeta_{\alpha} .
$$

Finally we introduce the assumption

$$
g^{\rho \sigma^{*} \zeta_{\rho, \sigma^{*}}}=0
$$

that is, 


$$
\Delta f=0 .
$$

This will also imply $g^{\rho \sigma^{*}} \eta_{\rho \sigma^{*}}=0$, and if for the sake of a later application we interchange the variables $\left(z_{\alpha}\right)$ and $\left(\bar{z}_{\alpha}\right)$ we obtain the following lemma.

LEMMA 9. If on a compact space with positive Ricci curvature a (selfadjoint) vector field $\zeta_{i}$ has the property that in the neighborhood of every point the components $\zeta_{\alpha}$. can be represented in the form

$$
\zeta_{\alpha^{*}}=\frac{\partial f}{\partial \bar{z}_{\alpha}} \text { with } \quad \Delta f=0
$$

then $\zeta_{\alpha^{*}} \equiv 0$, that is, $f$ is complex analytic.

If the curvature is only non-negative then $\zeta_{\alpha^{*}, i}=0$, that is, the derivatives $\partial f / \partial \bar{z}_{\alpha}$ are not necessarily 0 but have covariant derivative 0 .

Finally we shall have the following theorem.

THEOREM 6. If a compact space with positive Ricci curvature is subdivided into simplices $S_{1}, \cdots, S_{t}$, if for each $r, 1 \leqq r \leqq t$, there is defined a meromorphic functional element $\phi_{r}\left(z_{1}, \cdots, z_{n}\right)$ in some neighborhood $U_{r}$ of the closure of $S_{r}$, and if each difference $\phi_{p}-\phi_{q}$ is holomorphic in the intersection of $U_{p}$ and $U_{q}$ (whenever not empty), then there exists one meromorphic function $\Phi\left(z_{1} \cdots z_{n}\right)$ in $S$ such that, for each $U_{r}$, the difference $\Phi-\phi_{r}$ is holomorphic.

Proof. The proof proceeds as follows. We replace each $\phi_{r}$ in $U_{r}$ by another function $f_{(r)}$ which has no singularities but which on the other hand may also depend on the conjugate values $\bar{z}_{\alpha}$. In other words, $\partial f_{(r)} / \partial \bar{z}_{\alpha}$ need not be not equal to 0 . However, each $f_{(r)}$ shall be a solution of the Laplacean $\Delta f=0$, and the difference $f_{(p)}-f_{(q)}$ shall be equal to $\phi_{p}-\phi_{q}$ for all $(p, q)$. If such functions $f_{(r)}$ can be found then the components

$$
\zeta_{\alpha^{*}}=\frac{\partial f_{(r)}}{\partial \bar{z}_{\alpha}},
$$$$
\alpha=1, \cdots, n,
$$

are uniquely defined in $S$, and Lemma 9 applies immediately. Thus, for positive curvature, the components all vanish. In other words, the functions $f_{(r)}$ are automatically analytic, and the expression

$$
\Phi=\phi_{p}-f_{(p)}=\phi_{q}-f_{(q)}
$$

will define a meromorphic function in $S$. A similar proof holds for nonnegative curvature. Therefore the only remaining task is the construction of the functions $f_{(r)}$. This is a problem in potential theory 
and has a solution in either real or Hermitian space. We shall only indicate its solution since an elaboration of details would lead us far afield.

Suppose we are dealing with a compact $m$-dimensional space with a real positive fundamental form. We set up the Laplacean, and it is known ${ }^{12}$ that there always exists a Green's function $G(x ; \xi)$. It has the proper type of singularity for $x=\xi$, and it satisfies the relation

$$
\Delta_{x} G(x ; \xi)=-1 \text {. }
$$

Let $S_{r}$ and $U_{r}$ have the same meaning as in the theorem and let $B_{p q}$ denote the $(n-1)$-dimensional face separating $S_{p}$ and $S_{q}$, if such a face exists. In the integral to follow each such face will appear twice with opposite orientations. Now, for each $r$, let $\phi_{(r)}$ be an "arbitrary" function in $U_{r}$ with "singularities." By this we mean that it need be defined and differentiable only in some subset of $U_{r}$. However, the differences

$$
\phi_{p q}=\phi_{p}-\phi_{q}
$$

shall be each defined and differentiable in some neighborhood of the closure of $B_{p q}$. We now set up the Cauchy-Green integral

$$
\frac{1}{\omega} \sum_{p, q=1}^{t} \int_{B_{p q}} \sum_{\mu=1}^{m}\left(\phi_{p q}(\xi) \frac{\partial G(x, \xi)}{\partial \xi_{\mu}}-G(x, \xi) \frac{\partial \phi_{p q}(\xi)}{\partial \xi_{\mu}}\right) \sigma^{\mu} .
$$

The symbol $\sigma^{\mu}$ denotes a certain invariant $(m-1)$-dimensional external differential form, which in the Euclidean case is

$$
(-1)^{\mu} d \xi_{1} \cdots d \xi_{\mu-1} d \xi_{\mu+1} \cdots d \xi_{m}
$$

whereas $\omega$ is a numerical constant. For variable $x$ the integral as it stands defines a function in the interior of each $S_{r}$. Denoting this function by $f_{(r)}(x)$, we can show that it can be continued beyond $S_{r}$ through the interior of each $(m-1)$-dimensional face, that $f_{(p)}(x)$ $-f_{(q)}(x)$ has the value $\phi_{p q}(x)$ on $B_{p q}$, and that it is a solution of the Laplacean. ${ }^{13}$ All this would be true for arbitrary functions $\phi_{p q}(\xi)$ on $B_{p q}$, but then $f_{(r)}(x)$ need not be continuable through the interior of the boundary faces of dimension not greater than $m-2$. If however the prescribed saltus function has the special form (69) then $f_{(r)}(x)$ can be continued into an entire neighborhood of the closure of $S_{r}$, as needed.

In our complex space with a Laplacean of Hermitian type the

12 Compare, for instance, S. Bochner, Analytic mapping of compact Riemann spaces into Euclidean space, Duke Math. J. vol. 3 (1937) pp. 339-354.

${ }^{13}$ See the paper quoted in footnote 3, p. 657. 
existence of the Green's function could be proven by an adaptation of the proof for the real case. However the duplication can be avoided, and the complex case can be directly reduced to the real case, if we make the additional assumption on $S$ that it is basically a real space which in some of its allowable coordinates is also Hermitian. By this we mean that there exists a $2 n$-dimensional Riemannian space $S_{0}$ which is in topological correspondence with $S$. Each allowable coordinate system in $S$ is in correspondence with some coordinate system of $S_{0}$, and the Laplacean on $S$ is a transcription into complex parameters of the real Laplacean as defined on $S_{0}$.

12. Subspaces of Euclidean space. The following statements are of a local character. Suppose we are given several analytic covariant vector fields in a neighborhood of $n$ complex parameters. We denote them by $\zeta_{k / \alpha}$, where the subscript $k$ identifies the vector, and we attempt to define a fundamental tensor by the formula

$$
g_{\alpha \beta^{*}}=\sum_{k=1}^{l} \zeta_{k / \alpha} \zeta_{k / \beta^{*}}
$$

Our first concern must be to secure restriction (53). The obvious way of securing it is to assume that we have

$$
\zeta_{k / \alpha}=\frac{\partial \phi_{k}}{\partial z_{\alpha}}
$$

where $\phi_{k}(z)$ is an analytic scalar, because in this case

$$
g_{\alpha \beta^{*}}=\frac{\partial^{2}}{\partial z_{\alpha} \partial \bar{z}_{\beta}}\left(\sum_{k}\left|\phi_{k}\right|^{2}\right) .
$$

This is a crude way of securing (53) and there probably is some more elaborate condition; we shall deal with it nevertheless, since geometrically it corresponds to a mapping of our $z$-neighborhood into a $W$ neighborhood by the functions $W_{k}=\phi_{k}(f)$.

Theorem 7. If

$$
W_{k}=\phi_{k}\left(z_{1} \cdots z_{n}\right), \quad k=1, \cdots, l,
$$

is a parametrization of a subspace of Euclidean space, then its Hermitian tensor

$$
g_{\alpha \beta^{*}}=\sum_{k=1}^{l} \frac{\partial \phi_{k}}{\partial z_{\alpha}} \frac{\partial \bar{\phi}_{k}}{\partial \bar{z}_{\alpha^{*}}}
$$

generates a negative Ricci curvature. 
Proof. If we evaluate the determinant $G$ of $g_{\alpha \beta^{*}}$ then we find for its value the expression

$$
\sum_{p=1}^{C_{1, k}} D_{p} \bar{D}_{p}
$$

where each $D_{p}$ is the value of some $n$-dimensional determinant of the $n$ by $l$ matrix

$$
\left|\frac{\partial \phi_{k}}{\partial z_{\alpha^{*}}}\right|, \quad \alpha=1, \cdots, n ; k=1, \cdots, l .
$$

We now put

$$
R_{\alpha \beta^{*}}=\frac{\partial^{2} \log G}{\partial z_{\alpha} \partial \bar{z}_{\beta}}
$$

and form the sum

$$
-R_{\alpha \beta} \lambda^{\alpha} \overline{\lambda^{\beta}}
$$

for an arbitrary complex number $\lambda^{\alpha}$. Now, if we carry out the differentiations and if we put

$$
\eta^{p}=\frac{\partial D^{p}}{\partial z_{\alpha}} \lambda^{\alpha},
$$

then it turns out that the sum (71) has the value

$$
G^{-2} \sum_{p, q}\left|\eta^{p} D^{q}-\eta^{q} D^{p}\right|^{2}
$$

and this is non-negative. Also, if the matrix (71) has rank $n$, this must be strictly positive for some $\lambda^{\alpha}$, as claimed.

For the positiveness of (71) it is not necessary to assume that our vectors have the special form (70); however in general the matrix (73) is not the Ricci tensor and has no immediate geometrical interpretation.

13. Commutative analytic groups. We now assume the existence of contravariant analytic vectors $\zeta_{k}^{\alpha}$ and we set up the corresponding tensor

$$
g^{\alpha \beta^{*}}=\sum_{k=1}^{l} \zeta_{k \zeta k}^{\alpha \xi_{k}^{*}}
$$

If we denote its determinant by $H$, then by our last remark the matrix 


$$
-\frac{\partial^{2} \log H}{\partial z_{\alpha} \partial \bar{z}_{\beta}}
$$

is positive definite. If we introduce the inverse tensor $g_{\alpha \beta}$, then $G=H^{-1}$, and this would prove that the Ricci curvature is positive, provided $g_{\alpha \beta}{ }^{*}$ satisfies restriction (53). Now the identity

$$
g_{\beta \epsilon} e^{e^{* \alpha}}=\delta_{\beta}^{\alpha}
$$

implies

$$
\frac{\partial g_{\beta \epsilon^{*}}}{\partial z_{\gamma}} g^{\bullet^{*} \alpha}+g_{\beta \epsilon^{*}} \frac{\partial g^{\epsilon^{*} \alpha}}{\partial z_{\gamma}}=0
$$

or

$$
\frac{\partial g_{\beta \delta^{*}}}{\partial z_{\gamma}}=-g_{\beta e^{*}} \frac{\partial g^{\epsilon^{*} \alpha}}{\partial z_{\gamma}} g_{\alpha \delta^{*}}
$$

In applying (53) we hence obtain

$$
\frac{\partial g^{\epsilon^{*} \alpha}}{\partial z_{\beta}} g^{\beta \delta^{*}}=\frac{\partial g^{\delta^{*} \alpha}}{\partial z_{\beta}} g^{\beta \epsilon^{*}}
$$

which is equivalent with

$$
\sum_{p, q} \frac{\partial \zeta_{p}^{\alpha}}{\partial z_{\beta}} \overline{\zeta_{p}^{\alpha} \zeta_{q}^{\beta} \overline{\zeta_{q}^{\delta}}}=\sum_{p q} \frac{\partial \zeta_{q}^{\alpha}}{\partial z_{\beta}} \overline{\zeta_{q}^{\delta} \zeta_{p}^{\beta} \overline{\zeta_{p}^{\alpha}} .}
$$

The only obvious way of securing this relation is to assume that

$$
\frac{\partial \zeta_{p}^{\alpha}}{\partial z_{\beta}} \zeta_{q}^{\beta}-\frac{\partial \zeta_{q}^{\alpha}}{\partial z_{\beta}} \zeta_{p}^{\beta}=0
$$

for all $\alpha, p, q$. But this is the classical condition for commutative groups. Thus if the rank of the matrix $\left|\zeta_{p}^{\alpha}\right|$ is $n$, and the group operators are commutative, they give rise to an Hermitian metric with positive curvature.

Princeton University 